\title{
A COMMON GENERALIZATION OF LOCAL COHOMOLOGY THEORIES
}

\author{
by M. H. BIJAN-ZADEH
}

(Received 14 May, 1979)

1. Introduction. Throughout this note all rings considered will be commutative and noetherian and will have non-zero identity elements. $A$ will always denote such a ring and the category of all $A$-modules and all $A$-homomorphisms will be denoted by $\mathscr{C}_{A}$.

Let $\mathfrak{a}$ be an ideal of $A$. By the local cohomology functor w.r.t. a $[9, \S 2]$, denoted by $L_{a}$, we mean the functor from $\mathscr{C}_{A}$ to $\mathscr{C}_{A}$ whose object function is given by the formula:

$$
L_{\mathfrak{a}}(M)=\left\{x \in M \mid \text { there exists an integer } N>0 \text { such that } \mathfrak{a}^{N} x=0\right\},
$$

where $M$ is an $A$-module. Also, when $M$ and $N$ are $A$-modules and $f: M \rightarrow N$ is an $A$-homomorphism, $L_{\mathfrak{a}}(f)$ is the restriction of $f$ to the submodule $L_{\mathfrak{a}}(M)$ of $M$. It is easy to see that $L_{a}$ is an additive, covariant, $A$-linear, and left exact functor. For each $i \geq 0$, the $i$ th right derived functor of $L_{a}$ is denoted by $R^{i} L_{a}$.

The following generalization of local cohomology theory is given in [2]. Let $\Phi$ be a non-empty set of ideals of $A$. We call $\Phi$ a system of ideals (of $A$ ) if, whenever $\mathfrak{a}, \mathfrak{b} \in \Phi$, there is an ideal $\mathrm{c}$ in $\Phi$ such that $\mathrm{c} \subseteq \mathrm{ab}$. Now suppose $\Phi$ is a system of ideals of $A$. For every $A$-module $M$ we define

$$
L_{\Phi}(M)=\{x \in M \mid \mathfrak{a} x=0 \text { for some } \mathfrak{a} \in \Phi\}
$$

It is clear that $L_{\Phi}(M)$ is a submodule of $M$. Also for any homomorphism of $A$-modules $f: M \rightarrow N$, we define $L_{\Phi}(f): L_{\Phi}(M) \rightarrow L_{\Phi}(N)$ to be the restriction of $f$ to the submodule $L_{\Phi}(M)$ of $M$. Then $L_{\Phi}: \mathscr{C}_{A} \rightarrow \mathscr{C}_{A}$ is an additive, covariant, $A$-linear, and left exact functor. Moreover when $\Phi$ consists of only the powers of an ideal $a, L_{\Phi}$ is just the usual local cohomology functor w.r.t. a, $L_{\mathbf{a}}$. The functor $L_{\Phi}$ is called the general local cohomology functor w.r.t. $\Phi$. For each $i \geq 0$, the $i$ th right derived functor of $L_{\Phi}$ is denoted by $R^{i} L_{\Phi}$. In [2] it is shown that the study of torsion theories over $A$ is equivalent to studying this general local cohomology theory.

Another generalization of local cohomology functors has been recently given by $\mathbf{J}$. Herzog in [6]. For the moment let $A$ be local with unique maximal ideal $m$. For each $i \geq 0, H_{m}^{i}():, \mathscr{C}_{A} \times \mathscr{C}_{A} \rightarrow \mathscr{C}_{\mathrm{A}}$ is the functor defined by $H_{\mathrm{m}}^{i}(M, N)=\underset{\alpha}{\lim } \operatorname{Ext}_{A}^{i}\left(M / \mathrm{m}^{\alpha} M, N\right)$, $M, N \in \mathscr{C}_{A}$. Of course, for any ring $A$ and every ideal $a$ of $A$ we can define $H_{a}^{i}($,$) :$ $\mathscr{C}_{A} \times \mathscr{C}_{A} \rightarrow \mathscr{C}_{A}$ by the analogous formula. Then, by [9, (2.3)], $H_{a}^{i}(A$,$) and R^{i} L_{a}()$ are naturally equivalent functors (from $\mathscr{C}_{A}$ to $\mathscr{C}_{A}$ ).

The purpose of this note is to introduce a common generalization of these three theories. Let $\Phi$ be a system of ideals (of $A$ ) in the above sense. For each integer $i \geq 0$, we define the functor $H_{\Phi}^{i}():, \mathscr{C}_{A} \times \mathscr{C}_{A} \rightarrow \mathscr{C}_{A}$ by $H_{\Phi}^{i}(M, N)=\varliminf_{\mathfrak{a} \in \Phi} \operatorname{Ext}_{A}^{i}(M / \mathfrak{a} M, N), M, N \in$ 
$\mathscr{C}_{\mathrm{A}}$. Then $H_{\Phi}^{i}($,$) is an additive, A$-linear functor which is contravariant in the first variable and covariant in the second variable. These functors do indeed generalize all the functors described above. We shall see that most of the properties of general local cohomology functors given in [6] and [9] extend to these functors even under weaker conditions.

I wish to thank my supervisor, Dr. R. Y. Sharp, who suggested these ideas to me, and assisted me at all stages.

This work was supported financially by the University for Teacher Education of Iran.

2. Preliminaries. Throughout this section $\Phi$ will denote a system of ideals of $A$.

The functors $H_{\Phi}^{i}($, ) defined in Section 1 generalize functors studied in [1], [2], [6] and [9]:

(i) when $\Phi$ is taken as the powers of an ideal a say, then $H_{\Phi}^{i}($,$) is naturally$ equivalent to the functor $H_{a}^{i}($,$) as defined in [6];$

(ii) when $\Phi$ is taken as the powers of an ideal a say and $M=A$, then, by $[9,(2,3)]$, $H_{\Phi}^{i}(M$,$) and R^{i} L_{a}()$ are naturally equivalent functors (from $\mathscr{C}_{A}$ to $\mathscr{C}_{A}$ );

(iii) when $M=A$, then $H_{\Phi}^{i}(M$,$) is naturally equivalent with R^{i} L_{\Phi}()$ defined in [2]; we shall denote $H_{\Phi}^{i}(A$,$) simply by H_{\Phi}^{i}()$;

(iv) when $M=A$ and $\Phi=\{\mathfrak{a} \mid \mathfrak{a}$ is an ideal of $A, \operatorname{dim} A / \mathfrak{a} \leq d\}$ for some integer $d \geq 0$, then $H_{\Phi}^{i}(M$,$) is naturally equivalent with the functor H_{d}^{i}()$ studied in [1]. (It is to be understood that the dimension of the zero $A$-module is -1 .) We shall use these natural equivalences in future without any further comment.

We shall need the following lemma.

(2.1) Lemma. Let $M^{\prime}$ be a fixed $A$-module. Then, for each $i \geq 0$, the functors

$$
H_{\Phi}^{i}\left(M^{\prime}, \text { ) and } \underline{\lim }_{\underline{a} \Phi} H_{\mathfrak{a}}^{i}\left(M^{\prime},\right)\right.
$$

(from $\mathscr{C}_{\mathrm{A}}$ to $\mathscr{C}_{\mathrm{A}}$ ) are naturally equivalent.

Proof. We must first explain the construction of the functor $\lim _{\mathfrak{a} \in \Phi} H_{\mathfrak{a}}^{i}\left(M^{\prime}\right.$, ). Let $\mathfrak{a}, \mathfrak{b} \in \Phi$ with $\mathfrak{a} \leq \mathfrak{b}$, i.e. $\mathfrak{b} \subseteq \mathfrak{a}$. Also let $\alpha \geq 1$ be an integer. Then the natural homomorphism $M^{\prime} / \mathfrak{b}^{\alpha} M^{\prime} \rightarrow M^{\prime} / \mathfrak{a}^{\alpha} M^{\prime}$ induces the homomorphism $\operatorname{Ext}_{A}^{i}\left(M^{\prime} / \mathfrak{a}^{\alpha} M^{\prime}, N\right) \rightarrow \operatorname{Ext}_{A}^{i}\left(M^{\prime} / b^{\alpha} M^{\prime}, N\right)$ for any integer $i \geq 0$ and any $A$-module $N$. Also, if $\alpha \leq \beta$, then the diagram

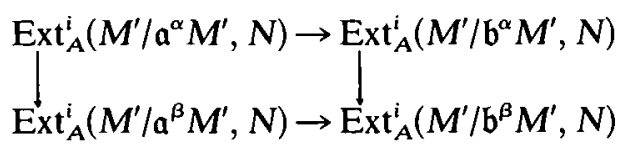

commutes. Thus we have a homomorphism

$$
\pi_{a}^{b}: \underset{\alpha}{\lim } \operatorname{Ext}_{A}^{i}\left(M^{\prime} / \mathfrak{a}^{\alpha} M^{\prime}, N\right) \rightarrow \underset{\alpha}{\lim } \operatorname{Ext}_{A}^{i}\left(M^{\prime} / \mathfrak{b}^{\alpha} M^{\prime}, N\right),
$$

that is

$$
\pi_{\mathrm{a}}^{\mathrm{b}}: H_{\mathrm{a}}^{\mathrm{i}}\left(M^{\prime}, N\right) \rightarrow H_{\mathrm{b}}^{\mathrm{i}}\left(M^{\prime}, N\right) .
$$


It is easy to see that these homomorphisms together with the modules $H_{a}^{i}\left(M^{\prime}, N\right)$ form a direct system of $A$-modules and $A$-homomorphisms over the directed set $\Phi$. Hence we may form the direct limit $\underset{a \in \Phi}{\lim } H_{a}^{i}\left(M^{\prime}, N\right)$.

Using the fact that for each integer $\alpha \geq 1$ and any ideal $b \in \Phi$ there is an ideal $c \in \Phi$ with $\mathfrak{c} \subseteq \mathfrak{b}^{\alpha}$, it is straightforward to see that, for each integer $i \geq 0$, the functors $H_{\Phi}^{i}\left(M^{\prime}\right.$, ) and $\underset{\mathfrak{a} \in \Phi}{\lim _{a}} H_{a}^{i}\left(M^{\prime},\right)$ are naturally equivalent.

3. Ring extension. Throughout this section $\Phi$ will denote a system of ideals of $A$. Suppose that $B$ is a second ring and that $f: A \rightarrow B$ is a ring homomorphism. For an ideal $\mathfrak{a}$ of $A$ we denote its extension to $B$ by $\mathfrak{a}^{e}$. Our main aim in this section is to prove a version of [6, (1.1.8)] in terms of systems of ideals. Then as a result of this we shall obtain $[6,(1.1 .8)]$ even under weaker conditions.

In what follows ()$^{A}: \mathscr{C}_{B} \rightarrow \mathscr{C}_{A}$ is the functor induced by restriction of scalars.

(3.1) Proposition. Let the situation be as above. Then

$$
\Phi^{f}=\left\{\mathfrak{a}^{e} \mid \mathfrak{a} \in \Phi\right\}
$$

is a system of ideals of $B$. Suppose that $M$ is an A-module. Then there is a homomorphism of connected right sequences of covariant functors (from $\mathscr{C}_{B}$ to $\mathscr{C}_{B}$ )

$$
\left(\psi^{i}\right)_{i \geq 0}:\left(H_{\Phi^{\prime}}^{i}\left(M \otimes_{A} B,\right)\right)_{i \geq 0} \rightarrow\left(H_{\Phi}^{i}\left(M,{ }^{A}\right)\right)_{i \geq 0}
$$

which is an isomorphism if $f$ is flat or $M$ is a f.g. projective A-module.

Proof. Let $S$ denote the $(A, B)$-bimodule $M \otimes_{A} B$ and let a be an ideal in $\Phi$. By [3, Ch. II, \$6]

$$
S / \mathfrak{a}^{e} S \cong\left(B / \mathfrak{a}^{e}\right) \otimes_{A} M .
$$

Also, it is easy to see that $B / a^{e} \cong B \otimes_{A}(A / \mathfrak{a})$. Hence, by $[3, C h$. II, §6] again, for any $B$-module $N$,

$$
\operatorname{Hom}_{B}\left(S / \mathfrak{a}^{e} S, N\right) \cong \operatorname{Hom}_{A}\left((A / \mathfrak{a}) \otimes_{A} M, N^{A}\right) .
$$

Moreover, by [3, Ch. II, §6], both (3.1.1) and (3.1.2) are functorial. Hence we deduce that $\operatorname{Hom}_{B}\left(S / a^{e} S,\right)$ and $\operatorname{Hom}_{A}\left((A / a) \otimes_{A} M,{ }^{A}\right)$ are naturally equivalent functors (from $\mathscr{C}_{B}$ to $\left.\mathscr{C}_{B}\right)$. This in turn yields the natural equivalence of functors

$$
\psi^{0}: H_{\Phi^{\prime}}^{0}\left(M \otimes_{\mathrm{A}} B,\right) \rightarrow H_{\Phi}^{0}\left(M,{ }^{A}\right)
$$

Since for any injective $B$-module $Q$ and each integer $i>0, H_{\Phi^{i}}^{i}\left(M \otimes_{A} B, Q\right)=0$, by Theorem 10 of Chapter 6 of [7], $\psi^{0}$ can be extended to a homomorphism of connected right sequences of covariant functors

$$
\Psi=\left(\psi^{i}\right)_{i \geq 0}:\left(H_{\Phi}^{i}\left(M \otimes_{A} B,\right)\right)_{i \geq 0} \rightarrow\left(H_{\Phi}^{i}\left(M,{ }^{A}\right)\right)_{i \geq 0} .
$$

Now suppose $B$ is flat as an $A$-module. Then, since $[11,(3.5)]$ any injective 
$B$-module remains injective when considered as an $A$-module, it follows by the corollary to Theorem 10 of Chapter 6 of [7] that the homomorphism $\Psi$ is an isomorphism of connected sequences of functors.

Finally suppose $M$ is a f.g. projective $A$-module. Hence $M$ is a direct summand of a f.g. free $A$-module. Since $[2,(2.5)(i)] R^{i} L_{\Phi}\left(N^{A}\right)=0$ for any injective $B$-module $N$ and each $i>0$ and the functor $H_{\Phi}^{i}\left(, N^{A}\right): \mathscr{C}_{A} \rightarrow \mathscr{C}_{A}$ is additive, it follows that $H_{\Phi}^{i}\left(M, N^{A}\right)=0$ for all $i>0$. The result follows.

(3.2) Corollary. Suppose $A$ and $B$ are local rings with maximal ideals $m$ and $r$ respectively. Let $f: A \rightarrow B$ be a ring homomorphism such that $\mathrm{m}^{e}$ is $\mathrm{r}$-primary. Also, let $M$ be an A-module. Then there is a homomorphism of connected right sequences of covariant functors $\left(\right.$ from $\mathscr{C}_{B}$ to $\left.\mathscr{C}_{B}\right)$

$$
\Psi=\left(\psi^{i}\right)_{i \geq 0}:\left(H_{\mathrm{r}}^{\mathrm{i}}\left(M \otimes_{\mathrm{A}} B,\right)\right)_{i \geq 0} \rightarrow\left(H_{\mathrm{m}}^{\mathrm{i}}\left(M,{ }^{\mathrm{A}}\right)\right)_{i \geq 0} .
$$

Moreover the homomorphism $\Psi$ is an isomorphism whenever $f$ is flat or $M$ is a f.g. projective A-module.

Proof. Take $\Phi=\left\{\mathrm{m}^{i} \mid i \geq 1\right\}$. Then $\Phi^{f}=\left\{\left(\mathrm{m}^{e}\right)^{i} \mid i \geq 1\right\}$. Hence, by Proposition (3.1), there is a homomorphism

$$
\Psi^{\prime}:\left(H_{\mathrm{m} f}^{i}\left(M \otimes_{\mathrm{A}} B,\right)\right)_{i \geq 0} \rightarrow\left(H_{\mathrm{m}}^{i}\left(M,{ }^{A}\right)\right)_{i \geq 0}
$$

of connected sequences of covariant functors (from $\mathscr{C}_{B}$ to $\mathscr{C}_{B}$ ). But since $\operatorname{rad}\left(\mathfrak{m}^{e}\right)=\mathrm{r}$, it is easy to see that there is an isomorphism of connected sequences of functors

$$
\Psi^{\prime \prime}:\left(H_{\mathrm{r}}^{\mathrm{i}}\left(M \otimes_{\mathrm{A}} B,\right)\right)_{i \geq 0} \rightarrow\left(H_{\mathrm{m}^{\prime}}^{i}\left(M \otimes_{\mathrm{A}} B,\right)\right)_{i \geq 0} .
$$

Hence there is a homomorphism of connected sequences of functors

$$
\Psi=\left(\psi^{i}\right)_{i \geq 0}:\left(H_{\mathrm{r}}^{i}\left(M \otimes_{A} B,\right)\right)_{i \geq 0} \rightarrow\left(H_{\mathrm{m}}^{i}\left(M,{ }^{A}\right)\right)_{i \geq 0} .
$$

Moreover by Proposition (3.1), $\Psi^{\prime}$ is an isomorphism whenever $f$ is flat or $M$ is a f.g. projective $A$-module. Hence $\Psi$ is an isomorphism whenever $f$ is flat or $M$ is a f.g. projective A-module.

Notation. Let $A$ and $B$ be local rings with maximal ideals $m$ and $r$, respectively. Also, let $k=A / m$ and $l=B / r$ be their residue fields. As in [6], for each $i \geq 0$, let $E_{A}^{i}()=,\operatorname{Hom}_{A}\left(H_{m}^{i}(),, E_{A}(k)\right)$ and $E_{B}^{i}()=,\operatorname{Hom}_{B}\left(H_{r}^{i}(),, E_{B}(l)\right)$ be the functors obtained from $H_{\mathrm{m}}^{\mathrm{i}}($,$) and H_{\mathrm{r}}^{\mathrm{i}}($,$) by cumposing with the appropriate Matlis duality$ functors.

(3.3) Corollary. With the above. notation suppose that $f: A \rightarrow B$ is a local homomorphism (i.e. $f(m) \subseteq r$ ) such that $B$ is a f.g. A-module by means of $f$. Also, let $M$ be a fixed A-module. Then there are natural transformations of functors

$$
\psi^{i}: E_{A}^{i}\left(M,{ }^{A}\right) \rightarrow E_{B}^{i}\left(M \otimes_{A} B, \quad(i \geq 0)\right.
$$

(from $\mathscr{C}_{B}$ to $\mathscr{C}_{B}$ ) such that $\psi^{i}$ is an equivalence whenever $f$ is flat or $M$ is a f.g. free A-module. 
(This corollary should be compared with Theorem (1.1.8) of [6].)

Proof. Since $B$ is f.g. as an $A$-module it is easy to see that $m^{e}$ is $r$-primary. Also it can be shown, by an argument similar to the proof of Theorem (4.2) of [11], that $\operatorname{Hom}_{\mathrm{A}}\left(B, E_{\mathrm{A}}(k)\right) \cong E_{\mathrm{B}}(l)$. The corollary now follows easily from Corollary (3.2).

4. Connections with Koszul complexes. In this section our aim is to state a general version of $[6,(1.1 .6)]$ which we shall need to use in the sequel. As we shall see, the condition that the module $N$ in $[6,(1.1 .6)]$ be finitely generated is superfluous. First of all we need to give some explanations.

Suppose that $\gamma_{1}, \gamma_{2}, \ldots, \gamma_{s}$ generate the ideal $\mathfrak{a}$, and that $M$ and $N$ are $A$-modules. Also, let $\alpha \leq \beta$ be positive integers. Then we can define the homomorphism

$$
\eta_{\alpha}^{\beta}: \operatorname{Hom}_{\mathrm{A}}\left(M /\left(\gamma_{1}^{\alpha}, \ldots, \gamma_{s}^{\alpha}\right) M, N\right) \rightarrow \operatorname{Hom}_{\mathrm{A}}\left(M /\left(\gamma_{1}^{\beta}, \ldots, \gamma_{s}^{\beta}\right) M, N\right)
$$

in a natural way so that the modules $\operatorname{Hom}_{A}\left(M /\left(\gamma_{1}^{\alpha}, \ldots, \gamma_{s}^{\alpha}\right) M, N\right)$ together with the maps $\eta$ form a direct system of $A$-modules and $A$-homomorphisms over $I$, the directed set of positive integers. In particular, the direct $\operatorname{limit} \underset{\alpha}{\lim } \operatorname{Hom}_{A}\left(M /\left(\gamma_{1}^{\alpha}, \ldots, \gamma_{s}^{\alpha}\right) M, N\right)$ can be formed. With this notation we have the following lemma.

(4.1) Lemma. The functors $\underset{\frac{\lim }{\alpha}}{\operatorname{Hom}} \operatorname{Hom}_{A}\left(M / \mathfrak{a}^{\alpha} M,\right)$ and $\underset{\alpha}{\lim } \operatorname{Hom}_{\mathbf{A}}\left(M /\left(\gamma_{1}^{\alpha}, \ldots, \gamma_{s}^{\alpha}\right) M,\right)$ are naturally equivalent (functors from $\mathscr{C}_{A}$ to $\mathscr{C}_{A}$ ).

Proof. The proof is straightforward and is therefore left to the reader.

Suppose that $M$ is a f.g. $A$-module and that $P$. is a projective resolution for $M$. Also, let $\mathfrak{a}=\left(\gamma_{1}, \gamma_{2}, \ldots, \gamma_{s}\right)$ be an ideal of $A$ and $\alpha \geq 1$ an integer. Let $K^{\alpha}$. denote the Koszul complex of $A$ w.r.t. the elements $\gamma_{1}^{\alpha}, \gamma_{2}^{\alpha}, \ldots, \gamma_{s}^{\alpha}$ of $A$. Here we use the notation and terminology about Koszul complexes of [8, Chapter 8]. In particular, every element of $K_{\mu}^{\alpha}$ $(0 \leq \mu \leq s)$ is uniquely expressible as a sum of elements of the form $e T_{i_{1}} T_{i_{2}} \ldots T_{i_{\mu}}$, where $e \in A, 1 \leq i_{1}<\ldots<i_{\mu} \leq s$ and $T_{1}, T_{2}, \ldots, T_{s}$ are new symbols. Next let $C_{*}^{\alpha}$ denote the single complex associated with the double complex $K_{*}^{\alpha} \otimes_{A} P$.. Now let $\alpha \leq \beta$ in $I$, and $N$ be an $A$-module. It is straightforward to see that the homomorphisms

$$
\begin{gathered}
K_{\mu}^{\beta} \rightarrow K_{\mu}^{\alpha} \\
e T_{i_{1}} \ldots T_{i_{\mu}} \mapsto \gamma_{i_{1}}^{\beta-\alpha} \ldots \gamma_{i_{\mu}}^{\beta-\alpha} e T_{i_{1}} \ldots T_{i_{\mu}}
\end{gathered}
$$

form a translation of (Koszul) complexes

$$
K_{\stackrel{\beta}{\beta}}^{\rightarrow} K_{\stackrel{*}{\alpha}} .
$$

This in turn induces a translation of double complexes

$$
K_{\bullet}^{\beta} \otimes_{\mathrm{A}} P . \rightarrow K_{\bullet}^{\alpha} \otimes_{\mathrm{A}} P .
$$

This induces a translation of single complexes

$$
C_{\bullet}^{\beta} \rightarrow C_{\bullet}^{\alpha} .
$$


But then we have the translation

$$
\operatorname{Hom}_{A}\left(C_{\bullet}^{\alpha}, N\right) \rightarrow \operatorname{Hom}_{A}\left(C_{\bullet}^{\beta}, N\right) \text {. }
$$

Hence we have the homomorphism of $A$-modules

$$
\pi_{\alpha}^{\beta}: H^{i}\left(\operatorname{Hom}_{A}\left(C_{\bullet}^{\alpha}, N\right)\right) \rightarrow H^{i}\left(\operatorname{Hom}_{A}\left(C_{\bullet}^{\beta}, N\right)\right) \quad(i \geq 0)
$$

It is easy to see that $\pi_{\alpha}^{\alpha}=$ id and if $\alpha \leq \beta \leq \eta$ in $I, \pi_{\alpha}^{\eta}=\pi_{\beta}^{\eta} \pi_{\alpha}^{\beta}$. Thus the modules $H^{i}\left(\operatorname{Hom}_{A}\left(C_{0}^{\alpha}, N\right)\right)(\alpha \in I)$ together with the homomorphisms $\pi$ form a direct system over $I$ so that the direct limit $\underset{\alpha}{\lim } H^{i}\left(\operatorname{Hom}_{A}\left(C_{\bullet}^{\alpha}, N\right)\right)$ may be formed.

One can generalize $[6,(1.1 .6)]$ to obtain the following theorem.

(4.2) TheOREM. Let the situation be as above. Then for any A-module N,

$$
H_{a}^{i}(M, N) \cong \underset{\alpha}{\lim } H^{i}\left(\operatorname{Hom}_{A}\left(C_{\bullet}^{\alpha}, N\right)\right) \quad(i \geq 0)
$$

5. Vanishing theorems. Throughout this section, $\Phi$ will denote a system of ideals of A. The following lemma will be needed later.

(5.1) Lemma. (See [12, (3.1)].) Suppose that a is an ideal of $A, M$ a non-zero, f.g. A-module of finite projective dimension, and $N$ an A-module of finite Krull dimension. Then $H_{\mathrm{a}}^{i}(M, N)=0$ for all $i>\operatorname{Pd}_{\mathrm{A}} M+\operatorname{dim} N$.

Proof. This can be established by induction on $\operatorname{Pd}_{A} M$. The case in which $\operatorname{Pd}_{A} M=0$ is an immediate consequence of $[9,(6.1)]$ and the additivity of the functor $H_{a}^{i}($, ). Suppose, inductively, that $s \geq 1$ and the assertion is true for every f.g. A-module of projective dimension less than $s$ and $M$ is a f.g. $A$-module of projective dimension equal to $s$. Hence there is an exact sequence of $A$-modules

$$
0 \rightarrow P_{s} \rightarrow P_{s-1} \rightarrow \ldots \rightarrow P_{1} \stackrel{\varepsilon_{1}}{\longrightarrow} P_{0} \stackrel{\varepsilon_{0}}{\longrightarrow} M \rightarrow 0
$$

where each $P_{i}(0 \leq i \leq s)$ is projective. Let $M^{\prime}=\operatorname{Im} \varepsilon_{1}$ so that the sequence

$$
0 \rightarrow M^{\prime} \rightarrow P_{0} \stackrel{\varepsilon_{0}}{\longrightarrow} M \rightarrow 0
$$

is exact. But then $\operatorname{Pd}_{A} M^{\prime}=s-1$. Let $X^{\prime}$. be a projective resolution for $M^{\prime}$ and $X^{\prime \prime}$. be a projective resolution over $M$. Then [7, Chapter 5, Theorem 16] there is a projective resolution, $X$. say, for $P_{0}$ which can be included in a split exact sequence

$$
0 \rightarrow X_{\bullet}^{\prime} \rightarrow X_{\bullet} \rightarrow X_{\bullet}^{\prime \prime} \rightarrow 0
$$

which is over (5.1.1). Let $K_{\bullet}^{\alpha}$ denote the Koszul complex for $A$ w.r.t. the elements $a_{1}^{\alpha}, a_{2}^{\alpha}, \ldots, a_{s}^{\alpha}$, where $a_{1}, a_{2}, \ldots, a_{s}$ generate the ideal $a$ and $\alpha \geq 1$ is any integer. Since both the tensor product and $\operatorname{Hom}_{A}(, N)$ are additive functors and (5.1.2) splits we get, for 
$\beta \geq \alpha$, a commutative diagram

$$
\begin{aligned}
& 0 \rightarrow \operatorname{Hom}_{A}\left(K_{\bullet}^{\alpha} \otimes_{A} X_{\downarrow}^{\prime \prime}, N\right) \rightarrow \operatorname{Hom}_{A}\left(K_{\bullet}^{\alpha} \otimes_{A} X ., N\right) \rightarrow \operatorname{Hom}_{A}\left(K_{\bullet}^{\alpha} \otimes_{A} X_{\bullet}^{\prime}, N\right) \rightarrow 0 \\
& 0 \rightarrow \operatorname{Hom}_{A}\left(K^{\beta} \otimes_{A} X_{\bullet}^{\prime \prime}, N\right) \rightarrow \operatorname{Hom}_{A}\left(K^{\beta} \otimes_{A} X_{.}, N\right) \rightarrow \operatorname{Hom}_{A}\left(K_{\bullet}^{\beta} \otimes_{A} X_{\bullet}^{\prime}, N\right) \rightarrow 0
\end{aligned}
$$

in which the rows are exact. The inductive step can now be completed with the aid of Theorem (4.2).

This completes the proof of the lemma.

(5.2) Proposition. Suppose that $M$ is a non-zero f.g. A-module of finite projective dimension and $N$ is an $A$-module of finite Krull dimension. Then $H_{\Phi}^{i}(M, N)=0$ for all $i>\operatorname{Pd} M+\operatorname{dim} N$.

Proof. By (2.1),

$$
H_{\Phi}^{i}(M, N)=\varliminf_{a \in \Phi}^{\lim } H_{a}^{i}(M, N)
$$

Now the proposition follows easily from Lemma (5.1).

Remark. Suppose that $M$ and $N$ are f.g. $A$-modules and that $M \otimes_{\mathrm{A}} N \neq 0$. Recall that the $N$-grade of $M$, written $\operatorname{grade}_{N} M$, is the length of any maximal $N$-sequence contained in $(0: M)_{A}$. Then grade $_{N} M$ is equal to the least integer $r$ such that $\operatorname{Ext}_{\mathrm{A}}^{r}(M, N) \neq 0$. For any ideal $\mathfrak{a}$ of $A$ for which $\mathfrak{a} N \neq N$ we define the $N$-grade of $\mathfrak{a}$ as $\operatorname{grade}_{N} A / \mathbf{a}$. For more details see $[10, \S 1]$.

Also, we put grade ${ }_{N} M=\propto$ if $M \otimes_{A} N=0$. We generalize this notion as follows.

(5.3) Definition. Let $\Phi$ be a system of ideals (of $A$ ), $M$ and $N$ f.g. A-modules. We define the $\Phi$-grade of $M$ w.r.t. $N$, denoted by $\Phi$-grade ${ }_{N} M$, as

$$
\Phi \text {-grade }{ }_{N} M=\inf _{\mathfrak{a} \in \Phi}\left\{\operatorname{grade}_{N}(M / \mathfrak{a} M)\right\} \text {. }
$$

Note: If, for every $a \in \Phi,(M / a M) \otimes_{\mathrm{A}} N=0$, then $\Phi$-grade ${ }_{N} M=\infty$; otherwise we have $\Phi$-grade ${ }_{N} M<\infty$.

(5.4) Lemma. Let $M$ and $N$ be f.g. A-modules and $\Phi=\left\{a^{i} \mid i \geqslant 1\right\}$ for some ideal a of A. Then

$$
\Phi-\operatorname{grade}_{N} M=\operatorname{grade}_{N}(M / a M) .
$$

In particular, when $A$ is local with maximal ideal $\mathrm{m}, M$ and $N$ non-zero and $\Phi$ consists of only the powers of $m$, then $\Phi$-grade ${ }_{N} M=\operatorname{depth}_{\mathrm{A}} N$.

Proof. It is enough to prove that for any integer $\alpha \geqslant 1$,

$$
\operatorname{grade}_{N}(M / \mathbf{a} M)=\operatorname{grade}_{N}\left(M / a^{\alpha} M\right) \text {. }
$$

But for any f.g. A-module $P$, the $N$-grade of $P$ is equal to the $N$-grade of the ideal $(0: P)_{A}$. Hence it is enough to show that

$$
\operatorname{grade}_{N}(0: M / \mathfrak{a} M)_{A}=\operatorname{grade}_{N}\left(0: M / a^{\alpha} M\right)_{A} \text { for all } \alpha \geqslant 1 .
$$


This follows easily by a straightforward argument and using the fact that for any ideal $\mathbf{b}$ of A,

$$
\operatorname{grade}_{N} \mathrm{~b}=\operatorname{grade}_{N} r(\mathrm{~b}) \text {. }
$$

(5.5) Proposrrion. Let the situation be as in Definition (5.3) above. Also, suppose that $\Phi$-grade ${ }_{N} M=t<\infty$. Then $H_{\Phi}^{\mathrm{i}}(M, N)=0$ for all $i<t$ and $H_{\Phi}^{t}(M, N) \neq 0$.

(Note that this generalizes $[\mathbf{1 2},(2.3)]$.)

Proof. We know that $H_{\Phi}^{i}(M, N)=\varliminf_{\underline{a} \in \Phi} \operatorname{Ext}_{A}^{i}(M / \mathfrak{a} M, N)$. Let $i<t$. Then we have $i<\operatorname{grade}_{N}(M / \mathfrak{a} M)$ for all $a \in \Phi$. This implies that $H_{\Phi}^{i}(M, N)=0$.

Next there is an ideal, $\mathfrak{b}$ say, in $\Phi$ for which $\operatorname{grade}_{N}(M / \mathfrak{b} M)=t$. Let $\mathfrak{a} \in \Phi$ be such that $\mathbf{b} \leqslant \mathfrak{a}$, i.e. $\mathfrak{a} \subseteq \mathbf{b}$. Since $\operatorname{grade}_{N}(M / \mathfrak{a} M) \geqslant t$, there is an $N$-sequence $x_{1}, x_{2}, \ldots, x_{t}$ which is contained in $\operatorname{ann}_{\mathbf{A}}(M / \mathfrak{a} M)$. Consider the natural epimorphism

$$
u: M / a M \rightarrow M / b M \text {. }
$$

Let $H=\operatorname{ker}(u)$ so that the sequence

$$
0 \rightarrow H \rightarrow M / \mathbf{a} M \rightarrow M / \mathbf{b} M \rightarrow 0
$$

is exact. This induces the long exact sequence

$$
\ldots \rightarrow \operatorname{Ext}_{A}^{t-1}(H, N) \rightarrow \operatorname{Ext}_{A}^{t}(M / b M, N) \rightarrow \operatorname{Ext}_{A}^{t}(M / \mathfrak{a} M, N) \rightarrow \ldots
$$

Since $\operatorname{ann}_{\mathrm{A}}(M / \mathrm{a} M) \subseteq \operatorname{ann}_{\mathrm{A}}(H)$ and $x_{1}, x_{2}, \ldots, x_{t}$ is an $N$-sequence contained in $\operatorname{ann}_{\mathrm{A}}(H)$, $\operatorname{Ext}_{A}^{t-1}(H, N)=0$. Thus for every $\mathfrak{a}$ in $\Phi$ with $\mathfrak{b} \leqslant \mathfrak{a}$, the map

$$
\operatorname{Ext}_{A}^{t}(M / \mathfrak{b} M, N) \rightarrow \operatorname{Ext}_{A}^{t}(M / \mathfrak{a} M, N)
$$

is a monomorphism. Since $\operatorname{Ext}_{A}^{t}(M / b M, N) \neq 0$, it follows that $\lim _{a \in \Phi} \operatorname{Ext}_{A}^{t}(M / a M, N) \neq 0$, as required.

\section{REFERENCES}

1. C. Bănică and M. Stoia, Singular sets of a module and local cohomology, National Institute for Scientific and Technical Creation/Institute of Mathematics, Bucharest, preprint.

2. M. H. Bijan-Zadeh, Torsion theories and local cohomology over commutative noetherian rings, J. London Math. Soc. (2) 19 (1979), 402-410.

3. H. Cartan and S. Eilenberg, Homological algebra (Princeton University Press, 1956).

4. S. Eilenberg and N. Steenrod, Foundations of algebraic topology (Princeton University Press, 1952).

5. A. Grothendieck, Local cohomology, Lecture Notes in Mathematics 41 (Springer-Verlag, 1967).

6. J. Herzog, Komplexe, Auflösungen und dualität in der Lokalen Algebra, preprint, Universität Essen.

7. D. G. Northcott, An introduction to homological algebra (Cambridge University Press, 1960). 

1968).

8. D. G. Northcott, Lessons on rings, modules and multiplicities (Cambridge University Press,

9. R. Y. Sharp, Local cohomology theory in commutative algebra, Quart. J. Math. Oxford (2) 21 (1970), 425-434.

10. R. Y. Sharp, Gorenstein modules, Math. Z. 115 (1970), 117-139.

11. R. Y. Sharp, Ramification indices and injective modules, J. London Math. Soc. (2) 11 (1975), 267-275.

12. N. Suzuki, On the generalized local cohomology and its duality, J. Math. Kyoto Univ. 18 (1978), 71-85.

Department of Pure Mathematics

UNIVERSITY OF SHEFFIELd

Hicks Building, Hounsfield Road

SHEFFIELd S3 7RH. 